\title{
Análise diagnóstica dos serviços de reabilitação que assistem vítimas de acidentes e violências em Recife
}

\author{
Diagnostic analysis of the rehabilitation services \\ that attend victims of accidents and violence in Recife
}

\author{
M aria Luiza Carvalho de Lima ${ }^{1,4}$ \\ Suely Ferreira Deslandes ${ }^{2}$ \\ Edinilsa RamosdeSouza ${ }^{3}$ \\ M aria Luiza Lopes Timóteo de Lima ${ }^{4}$ \\ AliceKelly Barreira ${ }^{4}$
}

${ }^{1}$ Departamento de

Medicina Social, U FPE. Av.

M oraes Rego s/n, Cidade

Universitária. 50670-420

Recife PE.

luiza@cpqam.fiocruz.br

${ }^{2}$ Departamento deEnsino,

Instituto Fernandes

Figueira, Fundação

Oswaldo Cruz.

${ }^{3}$ Centro Latino-Americano de Estudos de Violênciae

Saúde Jorge Carelli,

Departamento de

Epidemiologia e M étodos

Quantitativosem Saúde,

Escola Nacional deSaúde

Pública Sérgio Arouca,

Fundação Oswaldo Cruz.

${ }^{4}$ Departamento de Estudos

em Saúde Coletiva, Centro

de Pesquisas Aggeu

$M$ agalhães, Fundação

Oswaldo Cruz.
Abstract This paper aims to analyze the rehabilitation services in Recife, Brazil, confronting it with what the National Policy for Decreasing M orbimortality from Accidents and Violence (N PDM AV) professes. Six units of rehabilitation services were analyzed; five were from municipal administration and the other was an ON G on an agreement with SUS. A partial structured interview was conducted with the person in chargefor the Attention to the DisablePerson's $\mathrm{H}$ ealth aiming to map thenetwork. Also a questionnaire was applied to the managers of the six services aiming to identify the unit and its activities, structure and organization. The Rehabilitation Assistancenetwork in Recife do not meet the N PD M AV guidelines as it presents: decreased number of services/programs; lack of a multidisciplinary team; lack of technological support; deficient joint intra and inter sectors; little interaction between the victim and his family to reinsertion into family and social life; and still incipient prevention and promotion actions. In conclusion, there is an effort to adequate the actions to this population group; however there are relevant covering deficits of people, equipments, information and articulation registers among the various levels of this health network.

Key words Public policy, Rehabilitation, Accidents and violence
Resumo Este artigo visa analisar os serviços de reabilitação em Recife, confrontando com o que preconiza a Política Nacional de Redução de M orbimortalidade (PN M RAV). 0 estudo foi realizado em seis unidades/serviços de reabilitação, sendo cinco municipais e uma ONG conveniada ao SUS. A investigação desenvolveu-se com entrevista semi-estruturada destinada ao gestor da Atenção à Saúde de Pessoa com Deficiência, objetivando o mapeamento da rede, e questionário aplicado aos gerentes dos serviços para identificar as unidades e suas atividades, estrutura e organização desses serviços. A rede de atenção à reabilitação no município do Recife não cumpre satisfatoriamente as diretrizes da PN RM AV por apresentar número reduzido de serviços/programas; falta de uma equipe multidisciplinar; falta de aporte tecnológi co; deficiente articulação intra e intersetorial; pouca integração entre a vítima e a família para a reinserção familiar e social e incipientes atividades de prevenção e promoção. Conclui-se que há um esforço em adequar as ações a esse grupo populacional; no entanto, existem significativos dé ficits de cobertura, de pessoal, de equipamentos, de regi stro de informação e de articulação entre os vários níveis da rede de saúde.

Palavras-chave Política pública, Reabilitação, Acidentes e violências 
Introdução

As causas externas na cidade do Recife, desde a dé cada de noventa, vêm ocupando a segunda causa no padrão de mortalidade geral, representando 14,2\% do obituário geral em 2005 ejá se constituem na sexta maior causa de internação hospitalar ${ }^{1}$.

Dispõe-se de pou cos dados sobre as deficiências físicas e sequelas produzidas por esses agravos no contexto nacional e muito menos nos centros urbanos das capitais. 0 documento que apresenta a Política Nacional de Saúde da Pessoa Portadora deD eficiência menciona estudo realizado no Hospital das Clínicas (SP), com 174 pacientes com le são medular, identificando as seguintes causas: lesão traumática em $78 \%$ dos casos; ferimento de armas de fogo, 33\%; acidente de trânsito, 19,5\%; queda de altura, $19,5 \%$. A faixa etária mais atingida foi de 18 a 35 anos de idade, com $53 \%$ dos indivíduos, edestes, $81,7 \%$ do sexo masculino².

Outro estudo, da Rede Sarah (1997), identificou que, do total de 293 pacientes com traumatismo da coluna vertebral, $42 \%$ foram vítimas de acidentes de trânsito; $24 \%$, de disparo de armas de fogo; $12 \%$, de mergulhos em águas rasas; $11,6 \%$, de quedas e 9,5\%, de outros tipos de acidentes e violências. Da mesma forma, estudo internacional analisando pacientes portadores de lesão medular em área urbana de Houston, encontrou 183 pacientes, dos quais $57,9 \%$ eram paraplégicos e a etiologia mais frequente foi acidente de automóveis seguido por acidentes por armas de fogo? ${ }^{2}$.

Estes resultados ressaltam o impacto da violência por arma defogo e dos acidentes de trânsito nos agravos causadores de deficiências neste final de século, especialmente em centros urbanos.

N o Brasil, na área da saúde, a promulgação de políticas setoriais e de portarias vem sendo fundamental para a estruturação da atenção de reabilitação no setor público e no privado. D estacamos a Política Nacional de Redução da M orbimortalidade por Acidentes eViolências (PNRM AV) ${ }^{3}$, a Portaria do M inistério da Saúde $n^{\circ} 818^{4}$, que cria mecanismos para a organização e implantação de Redes Estaduais de Assistência à Pessoa Portadora de D eficiência Física; a Política Nacional de Saúde da Pessoa Portadora de D eficiência ea Política Nacional de Atenção às U rgências 5 .

A Política Nacional de Redução de M orbimortalidade por Acidentes e Violências orienta para a consolidação da atenção em reabilitação, a elaboração de normas técnicas e a necessidade de equipar as unidades de saúde para este fim. Propõe diretrizes como integrar a família do paciente no atendimento; prover equipe de recursos humanos multiprofissionais, na busca de evitar sequelas e incapacidades, e proporcionar condições para a reinserção na família, no trabalho, na sociedade e nos grupos culturais; oferecer recursos para reabilitação tais como órteses, próteses e meios delocomoção; promover mecanismos de informação, orientação e apoio que favoreçam o paciente e sua família; investir na sensi bilização degestores e profissionais de saúde a respeito de seu papel na orientação e na reintegração social dos portadores de sequelas e de seus familiares ${ }^{3}$.

$\mathrm{N}$ a perspectiva de criar mecanismos para a organização e implantação de Redes Estaduais de Assistência à Pessoa Portadora de D eficiência Física, em junho de 2001, a Portaria do M inistério da Saúde no 818 definiu quatro níveis de assistência à reabilitação física (serviços de reabilitação física primeiro nível dereferênciaintermunicipal; serviços dereabilitação física - nível intermediário; serviços de referência em medicina física e reabilitação; leitos de reabilitação em hospital geral ou especializado) para a estruturação e sistematização de uma rede hierarquizada possibilitando melhor a integralidade das ações.

Em todos os níveis de atenção, estão previstas normas técnicas eatribuições para prescrição, avaliação, adequação de treinamento, acompanhamento, dispensação de órteses, próteses e meio auxiliares de locomoção, preparação para alta, convívio social e familiar, além de orientação e suporte às equipes de Saúde da Família. Está preconizado que cada nível deve contar com o apoio de serviços auxiliares de diagnóstico e terapia, tais como os de patologia clínica, radiologia e ultrassonografia, compatíveis com sua complexidade.

A Portaria no 818 também destaca as seguintes diretrizes: promoção da qualidade de vida das pessoas portadoras de deficiências (com fortalecimento de iniciativas que conduzam à autonomia eà garantia de igualdade de oportunidades para as pessoas portadoras de deficiências); assistência integral a sua saúde (promovendo a ampliação da cobertura assistencial em reabilitação, incorporando tal assistência em unidades de diferentes níveis de complexidade); prevenção de deficiências; ampliação e fortal ecimento dos mecanismos de informação; organização e funcionamento dos serviços de atenção à pessoa portadora de deficiência; capacitação de recursos humanos, dentro de uma filosofia de formação contínua e de integralidade da atenção.

No primeiro nível de referência intermunicipal, devem ser desenvolvidas ações de prevenção primária e secundária, além de ações básicas de reabilitação. 0 documento determina que $a$ atenção secundária se volte para 0 atendimento das 
necessidades específicas advindas das incapacidades propriamente ditas, mediante atuação profissional especializada. E ressalta que o nível terciário deve promover 0 atendimento para as pessoas cujo tipo e gravidade das incapacidades requerem intervenção mais frequente e intensa.

Para acelerar o processo e reconhecendo o papel do setor na inclusão social, o Ministério da Saúde lançou, em 2003, a Política Nacional de Saúde da Pessoa Portadora de D eficiência4. Tal documento retoma os princípios garantidos na Constituição Brasileira quando afirma a competência comum à União, aos estados e aos municípios de cuidar da saúdee da assistência pública, dar proteção e garantia às pessoas portadoras de deficiências. Por sua vez, a Política Nacional de Atenção às Urgências ${ }^{5}$ orienta a inclusão do atendimento de reabilitação na própria comunidade, tendo como porta de entrada as unidades básicas de saúde, os hospitais de emergência ou os serviços de pronto atendimento, ondese deverealizar um diagnóstico precoce e avaliar as necessidades de cada pacientee dar o prosseguimento a seu acompanhamento.

0 presente artigo objetivou diagnosticar os avanços e lacunas existentes na implementação da atenção voltada para reabilitação à luz da Política Nacional de Redução de M orbimortalidade por Acidentes e Violências, aprofundando a análise da estrutura e organização desses serviços, no município do Recife.

\section{Métodos}

0 presente artigo constitui uma análise de parte dos dados da pesquisa "Análise diagnóstica de sistemas locais de saúde para atender aos agravos provocados por acidentes e violências", realizada no período de 2005 a 2006, que envolveu quatro capitais do Brasil e o Distrito Federal, dentre elas Recife, por ser uma das capitais com elevadas taxas de mortalidade por violência ${ }^{6}$.

0 desenho da investigação cumpre as características de um estudo transversal, descritivo. Utilizou-se a metodologia quanti-qualitativa, que se pautou nos princípios da triangulação de métodos, de modo a ampliar as possibilidades interpretativas. Para a triangulação de métodos, foram as estratégias: a) combinação ecruzamento demúltiplos pontos de vista; b) trabalho conjunto de pesquisadores com formação diferenciada; c) inclusão da visão de vários informantes; d) emprego de mais de uma forma de coleta de dados. Seu uso, na prática, permite interação, crítica intersubjetiva e comparação ${ }^{7-9}$.
As unidades/serviços de saúde incluídos no estudo foram mapeados segundo indicação do gestor municipal de Atenção à Saúde de Pessoa com Deficiência. Os serviços incluídos foram as cinco unidades municipais existentes euma unidadenão governamental (ONG) conveniada ao SUS, totalizando seis unidades. Foram excluídos os serviços de reabilitação estadual, por não serem referência para 0 atendimento da demanda da população em geral e sim para os egressos das enfermarias dos hospitais gerais ou dos ambulatórios de pacientes com sequelas das doenças crônico-degenerativas, como da rede particular conveniada, por serem de difícil acesso à população em geral. Estas seis unidades constituem as principais referências ao atendimento de reabilitação oferecido às vítimas de acidenteseviolência de Recife.

A investigação desenvolveu-se a partir dos procedimentos descritos a seguir. Inicialmente, foi aplicada uma entrevista semi-estruturada ao gestor municipal responsável pela Atenção à Saúde de Pessoa com D eficiência, com o objetivo de mapear e selecionar os serviçosa serem analisados. Em um segundo momento, aplicou-se o questionário aos gerentes dos serviços/programas identificados na fase anterior, contendo: 1) identificação da unidade; 2) caracterização das atividades de atendimento; 3 ) descrição da estrutura existente (equipe, instalações físicas erecursos financeiros); e 4) organização do serviço.

Também foi aplicada uma entrevista semi-estruturada destinada ao gestor responsável pela Política Municipal de Atenção à Saúde de Pessoa com D eficiência. Com esse roteiro, buscou-secompreender a ótica do gestor sobrea situação deatendimento das vítimas de acidentes e violências, ao cumprimento das diretrizes da PNRMAV e aos demais parâmetros de qualidade de atendimento preconizados nas políticas e portaria desse setor de atenção (reabilitação).

As entrevistas foram transcritas e interpretadas segundo os princípios da análise de conteúdo, a partir desua modalidadetemática. A análise de conteúdo priorizou a interpretação hermenêutica dos sentidoslatentese manifestosnos relatos, em detrimento de codificações estatísticas de sua ocorrência ${ }^{10}$.

A análise quantitativa usou a estatística descritiva (de frequências, medidas de tendência central e dispersão). 0 banco de dados foi digitado no Epi Info e analisado no Excel. Também foi realizada a crítica das variáveis.

A pesquisa foi aprovada no Comitê de Ética da Escola $\mathrm{N}$ acional deSaúde Pública e procurou atender as recomendações da resolução do CNS no $196 / 96$. 
Resultados

Dos seis serviços analisados, segundo a classificação especificada na Portaria no 818/M S que define os níveis de assistência segundo a sua complexidade, os cinco serviços pertencentes à secretaria municipal são serviços de primeiro nível e o da ONG foi classificado como de referência, com a particularidade de só atender crianças e adolescentes.

A Tabela 1 apresenta os principais tipos deatendimentos oferecidos pelos serviços estudados, em que todos os serviços oferecem a prevenção de sequelas e incapacidades ea avaliação e atendimento fisioterapêutico. Q uase todos os serviços realizam atendimento em grupo, estimulação psicomotora e avaliação médica, clínica e funcional. Quase não são realizados procedimentos como atendimento medicamentoso com dispensação deremédio, avaliação urodinâmica eatendimento cirúrgico.

Quanto à análise da composição da equipe de atendimento multiprofissional, como pode ser observado na Tabela 2, apesar da diversidade de profissionais, há uma predominância do fisioterapeuta (35\%), seguido por terapeuta ocupacional $(15 \%)$ e fonoaudiólogo ( $14 \%)$, especialidades importantes e que têm destaque na composição da equipe. Há um pequeno número de profissionais para oferecer suportepsicológico ( $11 \%)$ euma equipe mínima de enfermagem (12\%), somando enfermeiro, técnico eauxiliar.
Foi solicitado que cada serviço avaliasse a quantidade de profissionais de que dispõe para 0 atendimento a vítimas de violências eacidentes, atribuindo nota de zero a dez. Foi atribuída a nota três avaliando muito negativamente o número de especialistas e outros funcionários nos serviços. 0 insuficientenúmero demédico fisiatra (3) na composi ção das equipes dificulta a adequada avaliação da incapacidade e diagnóstico da parte clínica.

Ainda nesse item para avaliar a suficiência de equipes multiprofissionais, foi criado indicador que avalia a "proporção mínima de profissionais para as unidades primárias" indispensáveis para o seu funcionamento. Um dos serviços não alcança a equipe mínima de três profissionais, pois é preconizada no mínimo a existência de médico, fisioterapeuta ou profissional da área de reabilitação, assistente social e profissional de nível médio?.

$\mathrm{Na}$ Tabela 3, apresenta-se a avaliação da oferta de serviços às vítimas de violência e acidente. No que concerne ao acompanhamento pós-alta, referido em quatro das unidades, 0 atendimento domiciliar é realizado apenas em duas delas. Existem ações positivas sendo realizadas na maioria das unidades, como preparação para alta, suporte social e familiar e informações sobre os direitos das pessoas portadoras de deficiência.

Ainda em relação à estrutura dosserviços quanto ao provimento de órteses, próteses e meios auxiliares de locomoção, os serviços na sua maioria

Tabela 1. Distribuição dos serviços/programas em reabilitação, segundo tipo de atendimento realizado. Recife, 2006.

Tipo de atendimento Número de instituições

Atendimento em grupo 5

Prevenção de sequelas e incapacidades secundárias

Estimulação neuropsicomotora

Orientação familiar

Avaliação médica, clinica e funcional

Avaliação clínica especializada

Avaliação e atendimento em fisioterapia

Avaliação e atendimento em terapia ocupacional

Avaliação e atendimento em fonoaudiologia

Avaliação e atendimento em psicologia

Avaliação e atendimento em serviço social

Avaliação e atendimento em enfermagem

Avaliação e atendimento em nutrição

Atendimento medicamentoso com dispensação de remédios

Avaliação funcional e de diagnóstico de eletroneuromiografia e potenciais evocados

Avaliação urodinâmica

Atendimento clínico nas diversas especialidades médicas

Atendimento cirúrgico 
avaliam, prescrevem a utilização, mas apenas em dois serviços é realizada a dispensação.

Quanto a existência de recursos tecnológicos para o diagnóstico, apenas em duas unidades há recursos para análise clínica/laboratorial e radiologia convencional, uma unidade dispõe de ultrassonografia e medicina nuclear e urodinâmica são inexistentes em todos os serviços.

A organização dos serviços também foi aferida pela existência de rotinas e protocolos de atendimento às vítimas de violências e acidentes, acessível aos profissionais e foi mencionada em apenas duas unidades de serviços.

A articulação desses serviços com as equipes do PSF, assim como a comunicação com os serviços de atendimento pré-hospitalar e hospitalar, foi avaliada pelos gerentes das unidades como precária, revelando a desarticulação intrasetorial (entre

Tabela 2. Número e percentual de profissionais disponíveis nos serviços/programas de reabilitação. Recife, 2006.

\begin{tabular}{lrr}
\hline \multicolumn{1}{c}{ Profissionais } & Número & \multicolumn{1}{c}{$\%$} \\
\hline M édico fisiatra & 3 & 3 \\
Fisioterapeuta & 38 & 35 \\
Fonoaudiólogo & 15 & 14 \\
Enfermeiro & 4 & 4 \\
Assistente social & 7 & 6 \\
Psicólogo & 12 & 11 \\
Terapeuta ocupacional & 16 & 15 \\
Nutricionista & 3 & 3 \\
Farmacêutico & 3 & 3 \\
Responsável técnico & 4 & 4 \\
Técnico/auxiliar de enfermagem & 4 & 4 \\
Total & 109 & 100 \\
\hline
\end{tabular}

os três níveis de atendimento; pré-hospitalar, hospitalar e de reabilitação).

Foi solicitado aos respondentes que avaliassem diversos aspectos da assistência atribuindo uma nota de zero a dez a cada um dos itens avaliados. 0 transporte de pacientes recebeu nota quatro, o que revela um problema grave numa população que apresenta dificuldades de locomoção e escassos recursos financeiros.

As notas atribuídas para os itens existentes relativos ao atendimento às vítimas de acidentes/violências contemplaram de forma mais satisfatória a especialização da equipe $(7,8)$ e 0 registros do atendimento $(7,5)$. As notas menos satisfatórias foram para a comunicação com serviços de atendimento pré-hospitalar $(3,8)$ e transporte de pacientes $(4,8)$ (Tabela 4).

Analisou-se como os gestores avaliam a adequação do tempo desde a matrícula do paciente até 0 da evolução e da finalização da atenção às pessoas sequeladas por acidentes e violências. Observou-sequea maioria dos serviços considera que não apresenta boa adequação de tempo entre a lesão e a busca de tratamento e entre o tempo de-

Tabela 3. O ferta de serviços de reabilitação às vítimas de acidentes e violências. Recife, 2006.

\begin{tabular}{lc}
\hline \multicolumn{1}{c}{ Serviços/programas } & Número \\
\hline Atendimento domiciliar & 2 \\
Preparação para alta & 5 \\
Suporte para convívio social e familiar & 4 \\
Acompanhamento pós-alta & 4 \\
Informações sobre direitos das pessoas & 4 \\
portadoras de deficiências &
\end{tabular}

Tabela 4. Distribuição de serviços/programas, segundo as notas atribuídas para os seguintes itens existentes relativos ao atendimento às vítimas de acidentes/violências. Recife, 2006.

\begin{tabular}{lccc}
\hline & Mínimo & Média & Máximo \\
\hline Comunicação com serviços de atendimento pré-hospitalar & 0 & 3,8 & 8 \\
Comunicação com serviços de atendimento Hospitalar/emergência & 4 & 6,6 & 9 \\
Comunicação c/ serviços de atend. ambulatorial & 4 & 6,6 & 9 \\
Comunicação c/ serviços de equipes em Saúde da Família & 0 & 5,5 & 8 \\
Transporte de pacientes & 0 & 4,8 & 8 \\
Especialização da equipe & 5 & 7,8 & 10 \\
Instalações físicas do serviço & 1 & 6,3 & 10 \\
Registros do atendimento & 5 & 7,5 & 10 \\
Fluxos de referência e contra-referência & 5 & 7,0 & 10 \\
\hline
\end{tabular}


corrido para agendamento de primeira consulta, ou seja, existe fila de espera, retardo no atendimento, embora a permanência do pacienteno tratamento seja considerada adequada nas unidades.

\section{Discussão}

As lesões e traumas provocados por violências e acidentes têm gerado vários tipos de incapacidades, principalmentenosjovens, ese constituem em um desafio para os serviços de saúde do ponto de vista da sua estruturação e organização para assistir essa parcela da população. Frequentemente, as vítimas de violências eacidentes necessitam deuma atenção além do atendimento de emergência, incluindo cuidados hospitalares, reabilitação física e psicológica.

Na lógica de estruturação e organização do sistema de saúde, os serviços de nível primário devem existir em maior número na tentativa de garantir 0 acesso à população, a proximidade geográfica para a população e a prevenção de agravos e sequelas. Os serviços de reabilitação analisados em Recife foram em número de seis, na sua maioria, de nível primário.

A portaria do M S/GM n- 818 descreve os serviços denível primário ou primeiro nível de referência intermunicipal como aqueles que devem dispor de instalações físicas apropriadas, equipamentos básicos para reabilitação e recursos humanos com especialização, formação e/ou capacitação na área de reabilitação, para 0 atendimento a pacientes com deficiências físicas que requerem cuidados dereabilitação, prevenção de deficiências secundárias e orientação familiar.

Em entrevista com o gestor municipal, a fala corrente é de que reabilitação e reinserção social são timidamente propostas e executadas na rede desaúdemunicipal.

A estrutura de atendimento dos serviços de reabilitação para atendimento às vítimas deacidentes e violências tem sido percebida como insuficiente para a demanda que o município vem apresentando, pois está em fase de organização no que diz respeito à atenção em reabilitação, embora seja bom destacar que o modelo é descentralizado. Há um serviço para cada distrito sanitário, exceto em um dos distritos sanitários. É bom também destacar queo número deequipamentos disponíveiséinsuficienteenão há diversidade de equipamentos, dificultando corresponder às necessidades (gestor de atenção à saúde depessoa com deficiência - Recife).

Sendo unidades de nível de complexidade primária, foi observado que esses serviços também realizam al gumas atividades que seriam mais apropriadas para o nível intermediário, tais como atendimento em grupo, avaliação médica/clínica efuncional, o que denota falta de padronização das atividades segundo as diretrizes da Portaria no 818/ MS. Tomando como base as atividades propostas para os diferentes níveis de complexidade, observou-se que em poucas unidades são realizadas orientação familiar, ação importante e que não demanda recursos materiais ou equipamentos e depende, sobretudo, de tecnologias de comunicação e de produção de vínculo.

A ONU recomenda, enquanto atribuição da reabilitação, a prestação dos seguintes tipos de serviços: a) diagnóstico einterven ção precoce; $b$ ) atendimento e tratamento por médico fisiatra e não apenas fisioterapeuta; c) acompanhamento multiprofissional por assistente social, psicólogo, e outros; d) treinamento em atividades da vida diária para manter a independência, mobilidade, comunicação, entre outros; e) fornecimento de suporte técnico especializado para mobilidade e outros; $f$ ) serviços educacionais especializados; g) serviços de reabilitação (inclusive orientação profissional, colocação em emprego aberto ou abrigado); h) acompanhamento após alta 5 .

Embora exista uma preocupação em desconcentrar e descentralizar os serviços por distritos sanitários, ainda não há uma hierarquização devido ao insuficiente número de serviços existentes e no distrito sanitário VI não há nenhum serviço de reabilitação nem da rede própria nem conveniada. A penas na unidade de referência ocorre a dispensação de medicamentos, o que aponta para um baixo investimento nessenível, uma vez queos serviços públicos atendem uma população de baixo poder aquisitivo.

Contudo, constatamos nessa avaliação, que os serviços de reabilitação estudados, a exemplo do que ocorre em outras localidades do Brasil, têm 0 alvo de ação ainda muito reduzido ${ }^{7}$. Estudo realizado em outros municípios mostra uma realidade semelhanteà encontrada em Recife, onde se observou uma rededeaten ção aquém das necessidades locais, eos poucos serviços existentes privilegiam o atendimento de pessoas com deficiências físicas. Essefoco se deve à visibilidade das alterações físicas no desempenho funcional do indivíduo que, em geral, vê sua independência para a realização de atividades relacionadas ao cuidado pessoal, à mobilidade, às atividades ocupacionais e profissionais comprometida, deixando de lado os aspectos da saúde mental nos quais esses pacientes estão comprometidos ${ }^{1}$.

As equipes profissionais das unidades de saúde estudadas compõem-se basicamente de fisiote- 
rapeutas; os profissionais como fonoaudiólogo, psicólogo e terapeuta ocupacional estão presentes em não mais do que dois por unidade de saúde e registra-se que foi observada a carência das demais especialidades.

Alguns profissionais mencionados, como por exemplo o farmacêutico, e não listados para os serviços descritos, podem pertencer às unidades de saúde como um todo, uma vez que os serviços de reabilitação podem ser lotados em um hospital ou em outra modalidade de serviço.

No primeiro semestre de 2005 foram realizados 51.276 procedimentos realizados por diversos profissionais, para vítimas desses agravos, nas seis unidades estudadas. A estimativa da população do Recife portadora de deficiência para 0 ano de 2005 foi de $242.408^{12}$.

Quando comparado com o total deatendimento realizado, admitindo que em um ano tenha sido realizado o dobro do semestre, a cobertura seria aproximadamente de $14 \%$ da população necessitada dessa atenção - bem aquém do necessário.

Ademais, o registro de atendimentos é realizado segundo o total de procedimentos por cada tipo de profissional, não há informação sobre o tipo de deficiência atendida, nem há um registro organizado que permita saber quantos pacientes possuem agravos decorrentes de acidentes ou violências. Isso dificulta o conhecimento da magnitude do problema e da demanda por reabilitação, informações necessárias para subsidiar o planejamento da política municipal às pessoas portadoras de deficiência.

Além dessa dificuldade reconhecida pelo próprio gestor municipal de atenção à saúde da pessoa deficiente, outras são apontadas.

Conhecemos as dificuldades para estar implantando as diretrizes da própria política nacional, pois a questão passa por uma decisão política que, por sua vez, necessita de um aporte financeiro substancial do governo federal. Também ainda temos que, para al cançar uma integralidade das ações, melhorar as articulações com os outros serviços existentes, definir protocolos assistenciais, fluxo de atenção, capacitação, edeum registro dos pacientes com deficiências. H oje, o sistemaSUS não tem um quadro com estas informações específicas para identificar esta população. Falta um sistema de informação adequado para atender a implantação e dificuldades desta política.
Por sua vez, os documentos governamentais destacam a necessidade de esforços conjuntos das instituições privadas, públicas e da sociedade civil, no sentido depromover o retorno do pacientepara o convívio social, reintegrando-o à sociedade e à esfera profissional.

A falta de recursos tecnológicos descritosimpede o estabelecimento de diagnósticos mais precisos e acompanhamento terapêutico adequado. Por exemplo, amedicina nuclear ajudana avaliação mais precisa dostecidos, facilitando verificar a gravidade do impacto das lesões. A urodinâmica é importantíssima na avaliação dos traumas raquimedulares (TRM ), de pacientes que apresentam bexiga neurogênica - incontinência einfecção urinária.

No quadro apresentado, falta a eletromiografia, que permite a avaliação da condução nervosa, muito importante na averiguação de doenças neurológicas periféricas, fraturas com lesão traumáticados nervos, distrofia simpática reversa dos distúrbios osteomoleculares (DORT). A radiologia convencional só permite averiguar ossos earticulações. Paralelamente à precariedade de recursos tecnológicos em algumas unidades analisadas, lembramos ainda a dificuldade que é encaminhar pacientes na rede pública de saúde. Todas essas deficiências comprometem tanto 0 atendimento em si como a qualidade do tratamento e do acompanhamento dispensado às vítimas de violências e acidentes.

As melhores notas atribuídas para os itens existentes relativos ao atendimento às vítimas de acidentes/violências foram as relacionadas à especialização da equipe de profissionais e aos registros do atendimento. Como se percebe, ambas questões mais ligadas ao desempenho e interesse individual do que oriundas da estrutura, gestão e articulação com outros serviços.

A atenção de reabilitação constitui atualmente um eixo crítico no que concerne à aten ção às vítimas de acidentes eviolências, demandando urgente e contínuo investimento. 0 reconhecimento da problemática como prioridade na reorganização do modelo assistencial no município do Recifetêm sido um desafio para a gestão municipal. E, igualmente, é quase nulo o investimento na meta de atuação integrada, para que a pessoa portadora de deficiência alcance o nível físico, mental e ou social adequado as suas necessidades ${ }^{4}$. 


\section{Colaboradores}

M LC Lima, SF Deslandes e ER de Souza trabalharam na concepção teórica, elaboração e redação do texto; M LLT de Lima eAK Barreira participaram da análise dos dados e redação final do texto.

\section{Agradecimentos}

Agradecemos às auxiliares de pesquisa, Mariana Guimarães e Socorro Caldas, pela contribuição na coleta dos dados.

\section{Referências}

1. Souza ER, Lima ML. Panorama da violência urbana no Brasil e suas capitais. Cien Saude Colet 2006; 11(Supl.):1211-1223.

2. Brasil. M inistério da Saúde. Política Nacional de Saúde da Pessoa Portadora de Deficiência. Brasília: M inistério da Saúde; 2003.

3. Brasil. M inistério da Saúde. Política Nacional de Redução de M orbimortalidade por Acidentes e Violências. Brasília: Ministério da Saúde; 2001.

4. Brasil. M inistério da saúde. M anual de Legislação em Saúde da Pessoa Portadora de Deficiência. Brasília: M inistério da Saúde; 2003.

5. Brasil. M inistério da Saúde. Política Nacional de Atenção às U rgências. Brasília: M inistério da Saúde; 2004.

6. Marrie NSM, Deslandes SF, M inayo M CS. Análise da implementação do atendimento em reabilitação. In: M inayo MCS, Deslandes SF, organizadoras. Análise diagnóstica da Política N acional de Saúde para Redução de Acidentes e Violências. Rio de Janeiro: Fiocruz; 2007. p. 171-192.

7. Denzin NK. The Research Act. Chicago: Aldine Publishing Company; 1973.

8. Brasil. Portaria MS/GM $n^{\circ} 818$, de 5 de junho de 2001. Organização e implantação de Redes Estaduais de Assistência à Pessoa Portadora de Deficiência Física. Diário Oficial da União 2001; 7 jun.

9. M inayo MCS, Sanches O. Quantitativo-qualitativo: oposição ou complementaridade? Cad Saude Publica 1993; 9:239-262.

10. M inayo MCS, Assis SG, Souza ER, organizadoras. Avaliação por triangulação de métodos. Abordagem de programas sociais. Rio de Janeiro: Fiocruz; 2005.

11. Bardin L. Análise de conteúdo. Lisboa: Edições 70; 1979.

12. Neri M, Pinto A, Soares W, Costilla H. Retratos da deficiência no Brasil. Rio de Janeiro: FGV/IBRE, CPS; 2003.

Artigo apresentado em 25/10/2007

Aprovado em 13/08/2008

Versão final apresentada em 29/10/2008 